\title{
Image restoration by simple adaptive deconvolution
}

\author{
W. Waniak \\ Astronomical Observatory of the Jagiellonian University, ul. Orla 171, 30-244 Kraków, Poland
}

Received February 28, 1995; accepted September 1, 1996

\begin{abstract}
A new version of an iterative scheme of deconvolution originally introduced by Richardson (1972) and Lucy (1974) is presented. This algorithm is based on the Maximum Likelihood principle and imposes additional constraints on the solution of the inverse problem. The main idea of the newly presented method is to link the number of Richardson-Lucy iterations with the local difference between the object profile in the input image and the noisy background. If this difference is of the order of the noise level, only very few iterations are performed, whereas if this difference is much greater than this level, the number of iterations attains its maximum assigned value. Thus, the number of iterations is used as a regularizer for the restoration. Due to this adaptive approach, the background noise is highly suppressed and the probability of restoration artefacts is seriously diminished. What is more, the quality of restored images (described by the Kullback-Leibler distance between deconvolved and original profile) for the adaptive iterative scheme increases in comparison with the original approach, whereas the mean number of iterations per one pixel is substantially reduced. Some examples of the deconvolution of one- and twodimensional profiles presenting advantages of the new algorithm are described. Photometric fidelity of both methods is also compared and the predominance of the adaptive approach is confirmed.
\end{abstract}

Key words: methods: data analysis — techniques: image processing

\section{Introduction}

Inverse problems are very frequent in modern astronomy, as in many other scientific and technical disciplines. They occur if unknown parameters of an object or a phenomenon are related to observed data and if one wants to estimate these parameters from the observed quantities, which, of course, can be additionally distorted by a stochastic process like noise. Such problems belong to the class of ill-posed problems for which the uniqueness of the solution cannot be established and the solutions are oversensitive to the input data perturbations (Hadamard 1923; Tikhonov \& Arsenine 1977). The discrete and linear version of the inverse problem (most frequently considered in astronomy) has the form:

$$
s_{i}=s_{i}\left(\sum_{j=1}^{M} A_{i j} t_{j}\right) \quad i=1 \ldots N
$$

where $t_{j}$ are unknown, searched parameters, $A_{i j}$ describe the transformation and $s_{i}$ represent observed data, which are random variables. They are in general any statistics of one's choice (e.g. Poissonian in the case of astronomical images) and of expectation values given by the bracketed term.

Such inverse problems occur in astronomy in numerous cases, including, e.g. mapping of emission line regions of AGN's (Mannucci et al. 1992), mapping of accretion discs in binary systems (Horne 1985; Baptista \& Steiner 1991), surface imaging of stars (Piskunov \& Rice 1993), mapping of active regions in cometary nuclei (Waniak 1994). If the observed quantities and searched parameters belong to the same data space and $N$ is equal to $M$ the inverse problem becomes an image restoration. A straightforward solution of Eq. (1) via matrix inversion (assuming that the observed data are equal to the expected values) exhibits a very strong instability, therefore a special treatment of the inverse problem is desired. Generally, the problem is solved by looking for the extreme of a function of the observed and the unknown parameters. Many forms of this function have been considered during the past few decades by researchers. A brief presentation may be found in Titterington (1985). One of the most useful methods of solving the inverse problem is the iterative algorithm introduced by Richardson (1972) and Lucy (1974). Consecutive iterations maximize the likelihood function

$$
\sum_{i=1}^{N} s_{i} \ln \sum_{j=1}^{M} A_{i j} \tau_{j}
$$

where $\tau_{j}$ denotes estimates of $t_{j}$. This function not only controls the discrepancies between observed data 
and transformed searched parameters $\sum_{j=1}^{M} A_{i j} \tau_{j}$ but also ensures that output values are not negative. The Richardson-Lucy iterative algorithm can lead to a relatively smooth result when one starts the iterations from a constant solution and performs only a limited number of iterations. Unfortunately, for an excessively increasing number of iterations noise existing in observational data is amplified and the probability of appearance of deconvolution artefacts substantially increases. Some improvement of the Richardson-Lucy algorithm may be attained by addition of the penalty prescription to the basic function, such as e.g. the Maximum Entropy Method (Lucy 1994) or wavelet transform (Starck \& Murtagh 1994).

\section{Iterative algorithm}

The condition of maximization of the likelihood function (see Eq. (2)) leads to the commonly known iterative algorithm (Richardson 1972 and Lucy 1974):

$\tau_{k}^{r}=\tau_{k}^{r-1}\left[\sum_{i=1}^{N} A_{i j}\left(s_{i} / \sum_{j=1}^{M} A_{i j} \tau_{j}^{r-1}\right)\right] \quad k=1 \ldots M .(3)$

This iterative scheme preserves the sum of the retrieved parameters $\tau_{k}$ at each stage of the procedure and starting from the constant profile $\tau_{k}=T / M$ (where $\left.T=\sum_{i=1}^{N} s_{i}\right)$ converges to the stable solution of the ML problem.

For astronomical images obtained with multi-channel receivers (like CCD's) real objects are situated on a commonly appearing background produced, for example, by night sky illumination. Although the incoming photons have Poissonian statistics, after their conversion into measured counts-per-pixel and the consecutive stages of image treatment (e.g. bias, dark current and flat-field reductions) the initial statistics is transformed. Unfortunately, the way in which this transformation is carried out is strongly dependent on so many different factors that it is hard to predict the statistics of the output signal. The problem of image restoration based on generalized stochastic models of the signal has been addressed by some researchers (e.g.: Snyder et al. 1993; Snyder et al. 1994).

Of course some Poissonian-like dependence between the local noise and the local signal should exist in the resultant image. If the local signal increases both the local noise and the local $S / N$ (signal to noise ratio) increase. Equation (1) ought to be rewritten taking into account the image background.

$s_{i}=s_{i}\left(\sum_{j=1}^{M} A_{i j}\left(o_{j}+b_{j}\right)\right) \quad i=1 \ldots N$ where the $o_{j}$ represent the true object and the $b_{j}$ are the background. Equation (4) is valid for the case when the PSF is produced by telescope optics and receiver.

As has been mentioned in the previous section, for an excessive number of iterations the noise existing in observational data is amplified. This is connected with the structure of Eq. (3). For image fragments where only noisy background exists, the ratio ofobserved data $s_{i}$ and convolved parameters $\tau_{j}^{r-1}$ obtained at the iteration step $r-1$ may attain an excessively high or low value and may be strongly unstable. After convolution with the transposed PSF (i.e. correlation) these values are indeed smoothed out but can be transferred to image fragments occupied by crucial objects, distorting the results of deconvolution. In this way, the image noise is amplified and serious artefacts appear. Thus, the restored image may substantially differ from the original one. If the local $S / N$ ratio increases, which occurs for objects substantially exceeding the background noise, the iterative procedure weakly amplifies the noise. The probability of the appearance of artefacts decreases and the restored image is close to the original one.

This suggests a simple but quite ad hoc approach to the problem of stabilizing the iterative scheme for an increasing number of iterations. This number should be subjected to the local $S / N$ ratio. A similar approach, connecting the local number of Richardson-Lucy iterations with the local smoothness of structures in the image, has been previously elaborated by White (1993).

For image regions with only a noisy background, very few iterations should be performed, whereas for objects with a relatively high $S / N$ ratio, the number of iterations should increase to the maximum value asserted by the operator.

The main steps of the proposed algorithm follow. For the whole image the first iteration is performed according to Eq. (3) beginning from a constant profile $\tau_{k}=T / M$. This iteration has a special meaning because it produces the correlation of the input profile with the PSF:

$$
\tau_{k}^{1}=\sum_{i=1}^{N} A_{i k} s_{i} \quad i=1 \ldots M
$$

For a symmetric PSF this correlation is identical with optimal filtering. For an asymmetric PSF assuming a noiseless case we have:

$$
\begin{gathered}
\tau_{k}^{1}=\sum_{i=1}^{M} B_{i k} t_{k} \quad i=1 \ldots N \\
B_{i k}=\sum_{j=1}^{N} A_{j i} A_{j k} \quad i, k=1 \ldots M
\end{gathered}
$$

where $\mathbf{B}$ is a symmetric matrix. As can be seen, the result of the first iteration is a convolution of the original unblurred image with a symmetric profile substantially broader than the original PSF. 
If one takes into account that, in general, resolution in the restored image (for the appropriate criterion see Lucy 1992) increases with an increasing number of iterations the dependence between the local number of iterations and the local signal should not be a smoothly and slowly increasing function, if resolution for regions of different signals is to be constant. It should rather be a switch between the minimum number of iterations for image regions of very low $S / N$ and the maximum number of iterations for image regions of $S / N$ exceeding some limit. On the other hand, this dependence should not be as discontinuous as the Heaviside step-function, if proper work of the iterative scheme is desired. A sigmoid function commonly used in neural networks computations has been chosen by the author. It may be treated as a smooth realization of the Heaviside step-function. The ratio of the local signal to the local background noise has been chosen as the independent variable.

$R_{i}=R_{0}+\operatorname{INT}\left[\left(R_{\max }-R_{0}\right) /\left(1+\exp \beta x_{i}\right)\right]$

$x_{i}=S-\left[\left(\tau_{i}^{1}-b_{i}\right) / \sigma_{i}\right]$

where $R_{i}, R_{0}$ and $R_{\max }$ are, respectively, the actual, initial (equal to 1) and maximum (settled by the operator) number of iterations, $b_{i}$ are the local background and $\sigma_{i}$ are the background noise. The dimensionless parameter $S$ defines the value of signal to noise ratio for which the inflection of the sigmoid function occurs. The parameter $\beta$ controls the slope of the sigmoid function. These two parameters should be set in such a way that for $\tau_{i}^{1}-b_{i}$ of the order of background noise the number of iterations is close to $R_{0}$ and for $\tau_{i}^{1}-b_{i}$ substantially greater (e.g. 10 times) than the background noise, the number of iterations attains $R_{\max }$. The symbol INT denotes a function which gives an integer number nearest to the actual value of the real variable. Use of Eq. (7) requires the knowledge of local background $b_{i}$ and the level of its noise. If the underlying background is relatively smooth, it may be estimated by a smooth surface characterized by a restricted number of parameters. In such cases, the two-output-channel Richardson-Lucy method may be used. It puts one constraint on the solution for regions with data that can be fitted by a smooth surface and another constraint for the remaining regions (White 1993). In many cases it produces better results than the original Richardson-Lucy method.

After computing the maximum number of iterations for each image point the Richardson-Lucy iterations described by Eq. (3) may begin. However, an additional constraint is placed on the result of each iteration (including the first one). The image values, less than the background level $b_{i}$ are set equal to this level. This data clipping introduces some bias but its value is not photometrically significant because of substantial filtering given by the first step of the iterative procedure (see Eqs. (5) or (6)). Since the iterative scheme modified in such a way does not conserve the sum of the image signal, a further renormaliza- tion of the image is required. After renormalization, some values may be once again less than the background level, so a consecutive data clipping and image renormalization should be repeated. This iterative procedure is continued until the actual sum of image signals is sufficiently close to $T$. If the desired limiting discrepancy is of the order of a millimagnitude, only a couple of iterations ought to be carried out with CPU time consumption many times smaller than for one iteration of the deconvolution procedure. The renormalization scheme described above may destroy the local flux conservation by some nonlinearity of the photometric behaviour of this algorithm. However, if the expectation value of the local background is properly established the photometric error produced by this algorithm is fully negligible.

If the actual number of Richardson-Lucy iterations $r$ is greater than $R_{i}$ for a given pixel, the ratio of observed signal $s_{i}$ and convolved profile $\tau_{j}^{r-1}$ obtained at the previous iteration step is arbitrarily set equal to 1.0, which means that for this pixel, full agreement between observed value and the PSF convolution of the resultant profile has been achieved. Then, the convolution with transposed PSF is made, but for pixels for which $r>R_{i}, \tau_{j}^{r}$ is set equal to $\tau_{j}^{r-1}$. Richardson-Lucy iterations are carried out until $r$ is equal to the maximum $R_{i}$ over the whole image.

This modified iterative scheme has the property of an adaptive behaviour. If the local signal is close to the background level, only very few (or even one) iterations are carried out and the resultant profile is as smooth as the input profile (or even substantially smoother). In this case the resolution is highly suppressed but for low $S / N$ ratio any attempt to perform a "strong" deconvolution using any known method leads to spurious results. On the other hand, if the difference between a local signal and background level is really greater than the background noise, the local maximum number of iterations rapidly increases to $R_{\max }$ and resolution achieves its maximum level (expected for this case). What is more, the quality of the images restored with the adaptive algorithm for objects with large $S / N$ ratio should be better than using the original scheme. It follows presented earlier in this section. An exceptional advantage of this scheme should be attained for original objects with linear dimensions less than those of PSF, which is connected with a relatively weak penalization of the solution. The advantage of the presented method over the basic Richardson-Lucy method is mainly due to its adaptivity. Whereas the original Richardson-Lucy algorithm converges attaining maximum likelihood, the adaptive version converges attaining a value of likelihood which is somewhat lower (due to additional constrains placed on the solution) but, still very close to its maximum value.

An additional benefit of this approach is a remarkable reduction of the mean number of iterations per pixel. Indeed, for pixels where the actual $r$ is less than $R_{i}$, convolution of $\tau_{j}^{r-1}$ with PSF and convolution of the resultant 
set of the ratios with transposed PSF are replaced by substitutions of appropriate values. Furthermore, due to its simplicity, the adaptive scheme should be relatively quick (less CPU time consuming) in comparison with the other, more complex approaches to the image restoration problem that have been proposed (Piña \& Puetter 1993; Starck \& Murtagh 1994; Terebizh \& Biryukow 1994).

\section{Examples}

In order to compare the results of deconvolution and the computational efficiency of the original Richardson-Lucy algorithm with the adaptive scheme tests for one- and two-dimensional profiles have been carried out. As the measure of quality of the deconvolved profile, the Kullback-Leibler distances (Titterington 1985) were used. The first one describes the agreement between the original profile before convolution and the estimate obtained from the iterative procedure. The second one describes the discrepancy between the convolved and noisy input image and that obtained by PSF convolution of the resultant profile. The Kullback-Leibler distance (KL distance) is very appropriate in this case as it is fully consistent with the ML principle. If two compared profiles are identical, the likelihood achieves its maximum value, thus the actual likelihood for differing profiles can be subtracted from this value. In such a way, the KL distance is always positive and it tends toward zero if the agreement between the compared profiles increases. At iteration stage $r$ the two KL distances are computed according to the following equations:

$$
\begin{aligned}
& h^{r}=\sum_{j=1}^{M} t_{j} \ln \left(t_{j} / \tau_{j}^{r}\right) \\
& H^{r}=\sum_{i=1}^{N} s_{i} \ln \left(s_{i} / \sum_{j=1}^{M} A_{i j} \tau_{j}^{r}\right) .
\end{aligned}
$$

As the first test, the deconvolution of a one-dimensional double Dirac profile convolved with a Gaussian profile with FWHM of 10 pixels and distorted by noise has been carried out. The distance between the Dirac tips was 6 pixels. The expectation value of background level was constant over the whole profile and equal to 100 counts. Figure 1 presents the original twin Dirac profile and its PSF convolved image degraded by Poissonian noise. The profiles obtained by the original iterative scheme and its adaptive modification after 500 iterations are also presented. The advantage of the adaptive approach is fully evident. Not only the resolution is substantially increased but also the background noise is highly suppressed compared with the original algorithm which creates noticeable artefact (appearing as a "wavy" structure). This artefact is a one-dimensional version of the ringing structure present in a two-dimensional deconvolved image of a stellar profile. Figure 2 shows the KL distances for

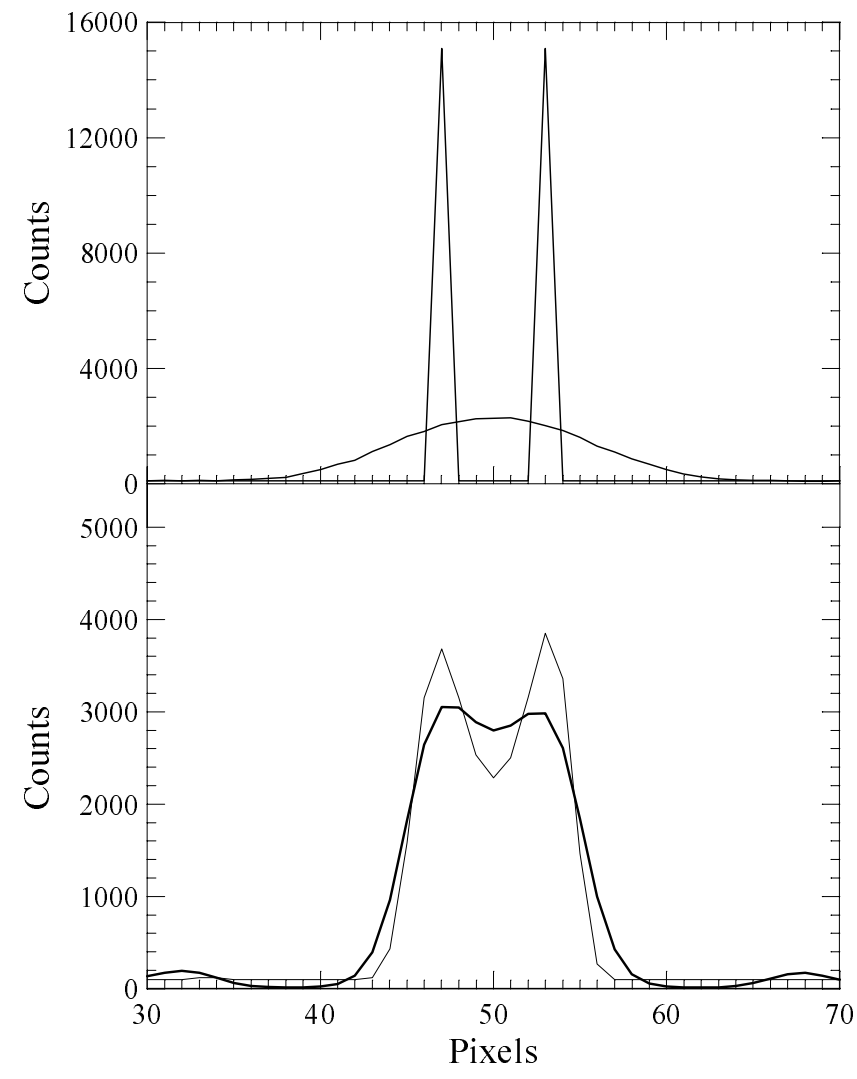

Fig. 1. Deconvolution of the double Dirac profile. Top panel - original profile and its image convolved with PSF and degraded by Poissonian noise. Bottom panel-deconvolved profile obtained using the Richardson-Lucy algorithm after 500 iterations (thick line) and that obtained by its adaptive version (thin line)

this test example. As has been previously reported by Lucy (1994), the computational efficiency of the original Richardson-Lucy scheme is satisfactory only for a few tens of iterations and then it quickly vanishes. On the contrary, for the modified algorithm, the efficiency retains a relatively high value. This means that for the adaptive approach the number of iterations may increase without any loss of the computational efficiency. Due to an additional penalization constraining on the ML solution by the adaptive algorithm, the KL distance $H$ tends to a small positive value (not to zero) and, additionally, does so more slowly than in the original scheme (see Fig. 2 bottom panel). As a two-dimensional example of image restoration the deconvolution of a double star image has been performed. Figure 3 presents the original image of a couple of pixels each having 20.000 counts imposed on the background of constant expectation value of 100 counts. The PSF profile comes from the real CCD observed stellar profile. The figure shows the image of a synthesized double star degraded by Poissonian noise and its deconvolved images after 100 iterations. KL distances are displayed in Fig. 4. The quality of restoration can be characterized by the total signal 


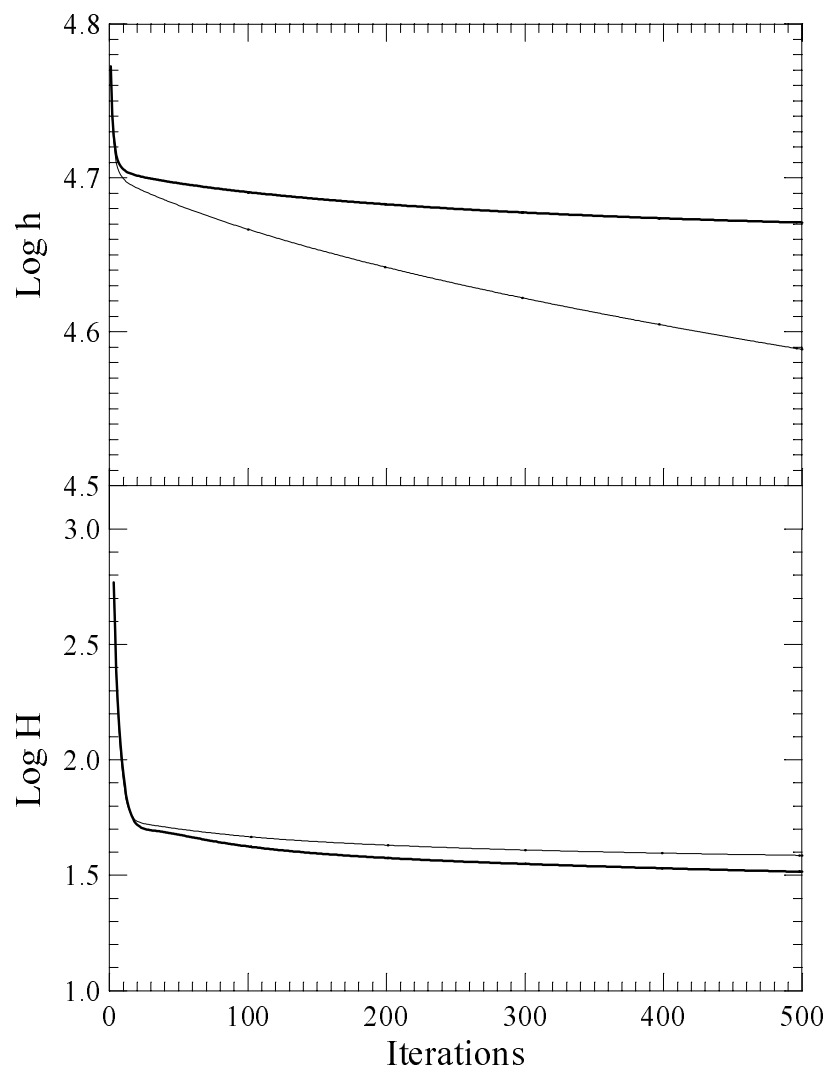

Fig. 2. Kullback-Leibler distances for profiles from Fig. 1 as functions of the number of iterations. Top panel-the distance between the deconvolved and original profile. Bottom panel - the distance between the input image and that obtained by convolving the restored profile with the PSF. Thick line - Richardson-Lucy algorithm, thin line - adaptive version

for the two central pixels of the deconvolved double star. For the original Richardson-Lucy iterative scheme $68 \%$ and for the modified algorithm $75 \%$ of the total energy is concentrated in these two pixels. Moreover, the background noise, which is amplified by the original algorithm, is fully suppressed by the adaptive one. For the original Richardson-Lucy iterative scheme the KL distance $h$ decreases to its minimum at 40 iterations, whereas for the modified algorithm it continuously decreases. This means that the original approach achieves its maximum computational efficiency after 40 iterations and subsequent iterations do not substantially improve the resultant profile. The adaptive scheme, on the contrary, retains its efficiency for an increasing number of iterations promising better image restoration.

\section{Photometric and astrometric properties}

One could assert that the newly proposed iterative algorithm, in some aspects established ad hoc, especially in the introduction of data clipping has photometric properties poorer than the original Richardson-Lucy

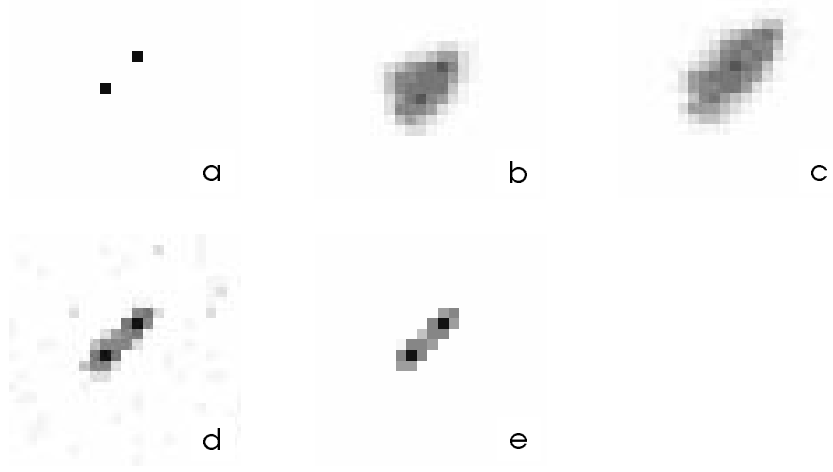

Fig. 3. Deconvolution of the double star profile (logarithmic grey scale). Panel a) original profile as a couple of pixels, b) observationally obtained PSF profile, c) synthesized double star blurred by Poissonian noise, d) image deconvolved by the Richardson-Lucy scheme after 100 iterations, e) profile restored using the adaptive algorithm

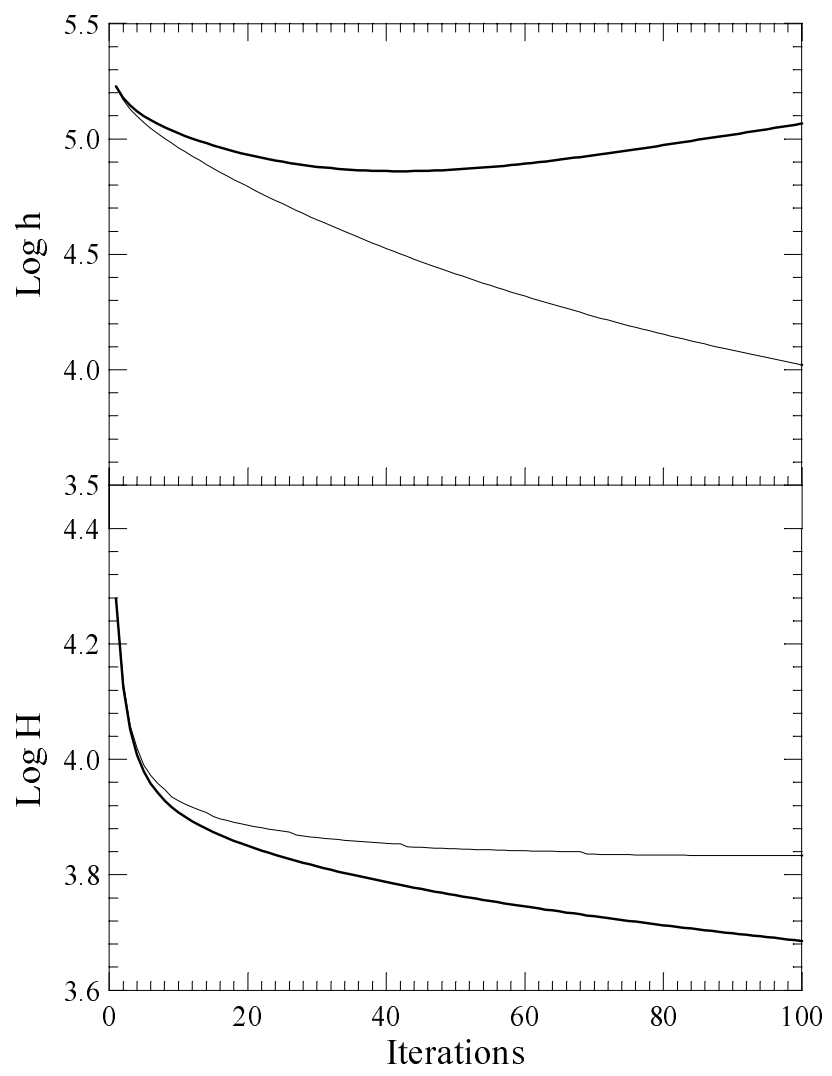

Fig. 4. KL distances for deconvolved images of the double star profile from Fig. 3 as functions of the number of iterations. For detailed description see Fig. 2 
algorithm. As a comparative test of photometric fidelity of both the deconvolution methods aperture photometry of synthetic stellar cluster has been undertaken. Two hundred stellar images having Gaussian profile of the radius at half-maximum equal to 2.0 pixel have been randomly distributed in the frame with a spatial concentration increasing toward the cluster centre. At least half of the stellar images were overlapping each other. Total counts contained in the stellar profile (stellar brightnesses) have been randomly distributed in the range of about 6 mag. The background was 16 counts. Seven different Poissonian noise realisations have been made for the basic frame. Each noisy frame has been restored using 250 iterations of both the methods. For the adaptive iterative scheme the gain in computational speed was 1.7 times. Then the aperture photometry with diaphragm radius of 2.0 pixel was carried out. Additionally, the astrometry using the stellar brightness centre was performed. As a measure of photometric fidelity the magnitude differences between the results of stellar aperture photometry and the original stellar brightnesses has been chosen. Figure 5 presents these differences $(\Delta \mathrm{mag})$ as the mean values in the set of seven different noise realisations. Generally, adaptive deconvolution has photometric properties markedly better than the Richardson-Lucy algorithm for which the ringing effect in connection with restoration artefacts produce distinct systematic errors. Astrometric properties of both algorithms are almost equally excellent. Mean deviations between original positions of stellar images and those obtained from restored frames are in both $X$ and $Y$ directions less than 0.2 pixel.

The results of the tests presented in the previous section and the comparison of photometric properties indicate the predominance of the newly elaborated adaptive approach over the basic algorithm, especially in the case of original objects with linear dimensions of the order of the FWHM of the PSF or less. Thus, the adaptive algorithm should be an excellent tool in restorations of crowded star fields enabling their precise photometry and astrometry. Its use for restoration of extended sources should always give some advantages in computational efficiency and quality of results over the original Richardson-Lucy scheme. As has recently been shown by Busko (1994), the Richardson-Lucy method is one of the most effective and accurate among deconvolution algorithms including the Maximum Entropy, CLEAN and Fourier-Wiener filtering. This means that any attempt of improving the basic algorithm is of great worth.

\section{Conclusions}

An adaptive version of the Richardson-Lucy iterative algorithm of deconvolution has been presented. This method using some additional constraints gives a solution very close to the one given by the Maximum Likelihood principle. The crucial point in this approach is spatially vary-

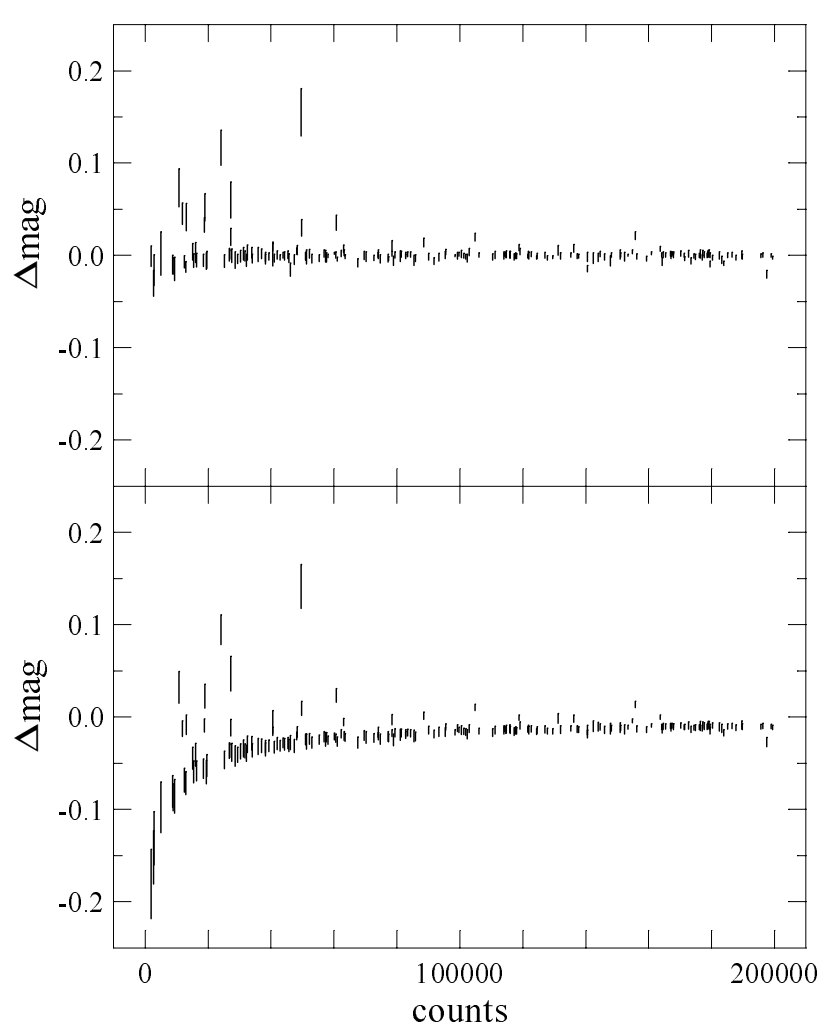

Fig. 5. Residuals between the magnitudes obtained from the aperture photometry of the restored frames and the original magnitudes of stars in the stellar cluster as a function of the original counts in stellar profile. Top panel - adaptive algorithm, bottom panel - Richardson-Lucy algorithm. Vertical bars represent standard deviations derived from the set of seven different noise realisations. Mean values of the residuals are at the centres of these bars

ing regularization achieved by a spatially varying number of iterations, contrary to the original scheme, where each image point is processed by the same number of iterations set by the operator. This leads to a spatially varying resolution. The number of iterations is controlled via the sigmoid function by the local difference between the object signal and the noisy background. When the difference between the object and the noisy background increases, the number of iterations also increases to attain its maximum value assigned by the operator. Due to this adaptivity, the background noise is highly diminished and artefacts of the restoration procedure almost vanish, which is not the case with the original Richardson-Lucy scheme. Moreover, in the new approach the mean number of iterations per one pixel is substantially less than the maximum value assigned by the operator.

To evaluate properties of both algorithms one- and two-dimensional profiles consisting of objects lying on the background were convolved with theoretical and observationally obtained PSF profiles and restored using both 
schemes. Photometric and astrometric properties of both methods have been evaluated from aperture photometry and brightness centre astrometry of the synthetic stellar cluster. The overall conclusion is that the computational efficiency of the modified iterative scheme is substantially greater, the quality of restored images and photometric fidelity substantially better than that of the original one. This is generally due to the adaptivity of the newly elaborated approach. Its predominance over the basic algorithm is especially evident in the case of the restoration of crowded star fields.

Acknowledgements. The author is very grateful to Dr. H.-M. Adorf and Dr. Roques, referees of this paper, for many helpful comments on an initial version of this work.

\section{References}

Baptista R., Steiner J.E., 1991, A\&A 249, 284

Busko I.C., 1994, PASP 106, 1310

Hadamard J., 1923, Lectures on the Cauchy Problem in Linear Partial Differential Equations. Yalle University Press, New Haven
Horne K., 1985, MNRAS 213, 129

Lucy L.B., 1974, AJ 79, 745

Lucy L.B., 1992, AJ 104, 1260

Lucy L.B., 1994, A\&A 289, 983

Mannucci F., Salvati M., Stanga R.M., 1992, ApJ 394, 98

Piña R.K., Puetter R.C., 1993, PASP 105, 630

Piskunov N.E., Rice J.B., 1993, PASP 105, 1415

Richardson W.H., 1972, J. Opt. Soc. Am. 62, 55

Snyder D.L., Hammoud A.M., White R.L., 1993, J. Opt. Soc. Am. A 10, 1014

Snyder D.L., Helstrom C.W., Lanterman A.D., Faisal M., White R.L., 1994, Proc. The restoration of HST images and spectra II, 18-19 Nov. 1993, Baltimore, MD 21218. In: Hanisch R.J. and White R.L. (eds.) STScI, p. 139

Starck J.L., Murtagh F., 1994, A\&A 288, 342

Terebizh V.YU., Biryukow V.V., 1994, Ap\&SS 218, 65

Tikhonov A.N., Arsenine V.Y., 1977, Solutions of ill-posed problems. Winston \& Sons, Washington DC

Titterington D.M., 1985, A\&A 44, 381

Waniak W., 1994, Icarus 111, 237

White R.L., 1993, in: Restoration: Newsletter of the STScI's Image Restoration Project, Hanisch B. (ed.), STScI, 18-19 Nov. 1993, Baltimore, MD 21218, p. 11 